\title{
IMPACTO DEL REUSO DE FRECUENCIA FRACCIONAL EN LA REDUCCIÓN DE INTERFERENCIA INTERCELDA EN LTE.
}

\author{
IMPACT OF FRACTIONAL FREQUENCY REUSE IN REDUCING INTER-CELL \\ INTERFERENCE LTE.
}

Betty Nayibe Fernández Campo; Lesly Alejandra González Camacho; Claudia Milena Hernández Bonilla.

$\begin{array}{r}\text { Grupo I+D Nuevas Tecnologías en Telecomunicaciones (GNTT), Universidad del Cauca. } \\ \text { bnfernandez@unicauca.edu.co, alejitagonzalez@unicauca.edu.co, claudiah@unicauca.edu.co. }\end{array}$
Recibido: 07 de febrero de 2014
Aceptado: 08 de marzo de 2014
*Correspondencia del autor. Grupo I+D Nuevas Tecnologías en Telecomunicaciones (GNTT), Facultad de Ingeniería Electrónica y
Telecomunicaciones, Universidad del Cauca, Popayán (Cauca). Email: claudiah@unicauca.edu.co.

\section{RESUMEN}

La Interferencia Inter-Celda (ICI, Inter-Cell Interference) es un problema que desafía el desempeño de las redes Evolución a Largo Término (LTE, Long Term Evolution), sin embargo existen técnicas de Coordinación de Interferencia Inter-Celda (ICIC, Inter-Cell Interference Coordination) como el Reuso de Frecuencia Fraccional (FFR, Fractional Frequency Reuse) que permiten mitigar dicha interferencia y mejorar el desempeño de los Equipos de Usuario (UE, User Equipment), especialmente aquellos terminales situados en el borde de la celda. Este artículo analiza el desempeño de la técnica Reuso de Frecuencia Fraccional (FFR) en LTE, en función de dos parámetros de configuración: Umbral de Relación Señal a Ruido más Interferencia (SINR, Signal to Interference plus Noise Ratio) y partición de Ancho de Banda (BW, Band Width). Se evalúa la capacidad e interferencia mediante diagramas de dispersión, curvas de Función de Probabilidad Acumulada Empírica (ECDF, Empirical Cumulative Density Function) y cálculos estadísticos.

Palabras claves: Desempeño, Eficiencia espectral, FFR, ICI, LTE, SINR, Throughput.

\begin{abstract}
The Inter-Cell Interference (ICI) is a problem that impacts the performance of Long Term Evolution (LTE) networks, however there are techniques like Inter-Cell Interference Coordination (ICIC) as Fractional Frequency Reuse (FFR) that mitigate the interference and improve the performance of User Equipment (UE), especially those terminals on the edge cell. This article focuses on the Fractional Frequency Reuse (FFR) performance in LTE, according two configuration parameters: Threshold Signal to Interference plus Noise Ratio (SINR) and partition Bandwidth (BW). Capacity and interference were evaluated by scatter plots, Empirical Cumulative Density Function (ECDF) curves and statistical calculations.
\end{abstract}

Keywords: ICI, FFR, LTE, Performance, SINR, Spectral Efficiency, Throughput. 


\section{INTRODUCCIÓN}

En el 2013 la industria de la telefonía móvil celular se ha incrementado en más de cuatro mil millones de suscriptores a nivel mundial (1). Esto ha llevado al desarrollo de nuevas tecnologías que permitan atender la creciente población de usuarios y su gran demanda de servicios (2).

El desarrollo de los sistemas de comunicaciones móviles ha pasado por sistemas analógicos $(1 \mathrm{G})$, digitales $(2 \mathrm{G})$, sistemas con transmisiones de datos limitados (3G) hasta los sistemas de hoy en día, entre los cuales Evolución a Largo Término (LTE) es una de las tecnologías de acceso dominante(1), capaz de garantizar mayores velocidades de transmisión y soporte de todos los servicios a través del Protocolo de Internet (IP, Internet Protocol) (2).

Evolución a Largo Término (LTE) es un estándar basado en la técnica de Acceso Múltiple por División de Frecuencia con Portadora Única (SC-FDMA, Single Carrier Frequency-Division Multiple Access) para el Enlace de Subida (UL, UpLink) y en la técnica de Multiplexación por División de Frecuencia Ortogonal (OFDM, Orthogonal Frequency Division Multiplexing) para el Enlace de Bajada (DL, DownLink), con lo cual permite tener mayor eficiencia espectral y menor latencia en una red de comunicaciones (3).

Gracias a OFDM, el ancho de banda del sistema se divide en un número de sub-portadoras ortogonales entre sí, que permite reducir el efecto de la Interferencia Intersimbólica (ISI, Inter-Symbol Interference) y de la Interferencia Interportadora (ICaI, Inter-Carrier Interference) dentro de la celda. Sin embargo, la Interferencia Co-Canal (CCI, Co-Channel Interference) o Interferencia Inter-Celda (ICI) es el principal problema de LTE, dado que emplea un factor de reuso de frecuencia igual a 1, donde las mismas subportadoras son utilizadas por diferentes usuarios en celdas adyacentes (4), lo cual afecta especialmente a los terminales situados en el borde de la celda, que reciben señales interferentes de las celdas vecinas, llevando a una degradación de su desempeño (5).

El Reuso de Frecuencia Fraccional (FFR) se plantea como una técnica de Coordinación de Interferencia Inter-Celda (ICIC), la cual se basa en un esquema de reutilización $n(n>1)$ (6), donde la celda se divide en 2 regiones, una interior cercana a la Estación Base (BS,
Base Station) y otra exterior situada en el borde de la celda; el ancho de banda se divide en 4 sub-bandas, donde 1 sub-banda es asignada a la región interior con un factor de reuso igual 1 y las otras 3 son asignadas al exterior de las celdas con un factor de reuso igual a 3 como se muestra en la figura 2, de esta forma, la Interferencia Intra-Celda (InCI, Intra-Cell Interference) se mitiga, y la Interferencia Inter-Celda (ICI) se reduce sustancialmente (7).

Este trabajo evalúa la técnica Reuso de Frecuencia Fraccional (FFR) en LTE, que garantiza un equilibrio entre la eficiencia espectral y la mitigación de la interferencia (8), reduce la probabilidad de interrupción del servicio para los usuarios del borde y mejora el desempeño del sistema (9).

\section{MATERIALES Y MÉTODOS}

La metodología empleada para diseñar, implementar y analizar la técnica de Reuso de Frecuencia Fraccional (FFR) en un sistema LTE, es una adaptación de la Metodología de Simulación de Sistemas de Telecomunicaciones, en la cual se tuvieron en cuenta los parámetros y variables más relevantes.

En la Figura 1 se presenta el diagrama de flujo del sistema con Reuso de Frecuencia Fraccional (FFR).

La herramienta empleada fue el simulador LTE de Viena a nivel de sistema, desarrollado por Josep Colom Ikuno, Martin Wrulich y Markus Rupp (10), (16) en el Instituto de Comunicaciones e Ingeniería de Radio-Frecuencia de la Universidad de Viena, Austria, bajo la licencia académica no comercial.

\section{Modelo del sistema}

El escenario considerado es una red LTE con celdas hexagonales desplegadas con un número constante de Equipos de Usuarios (UE) distribuidos aleatoriamente, empleando una configuración de antenas Única Entrada Única Salida (SISO; Single Input and Single Output) y planificadores Round Robin y Proportional Fair.

En la Figura 2, se muestra que cada Nodo B Evolucionado (eNB, Evolved Node B) contiene 3 celdas en forma hexagonal, cada una se divide en una zona centro denominada zona de Reuso Completo (FR, Full Reuse) y una zona borde denominada zona de Reuso Parcial (PR, Partial Reuse). En las zonas de Reuso 


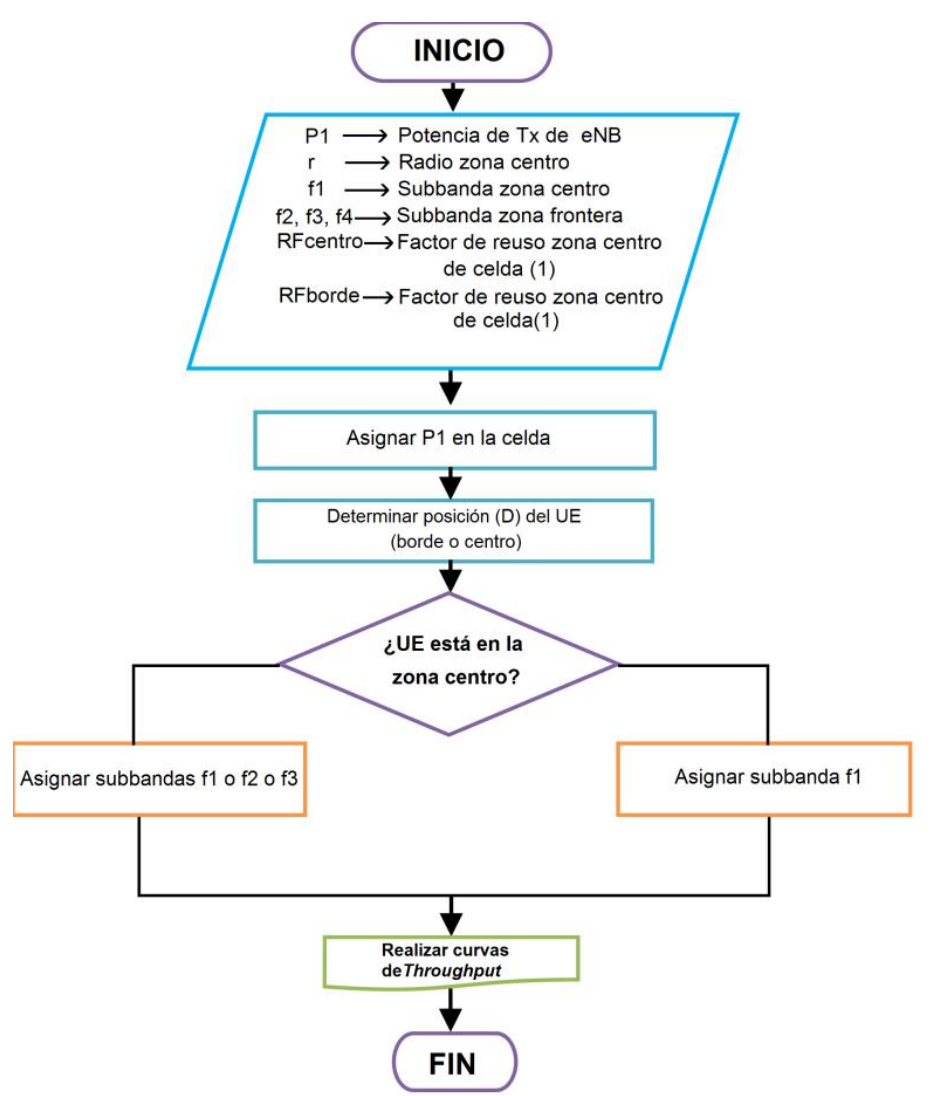

Figura 1. Diagrama de Flujo de la Técnica Reuso de Frecuencia Fraccional (FFR).

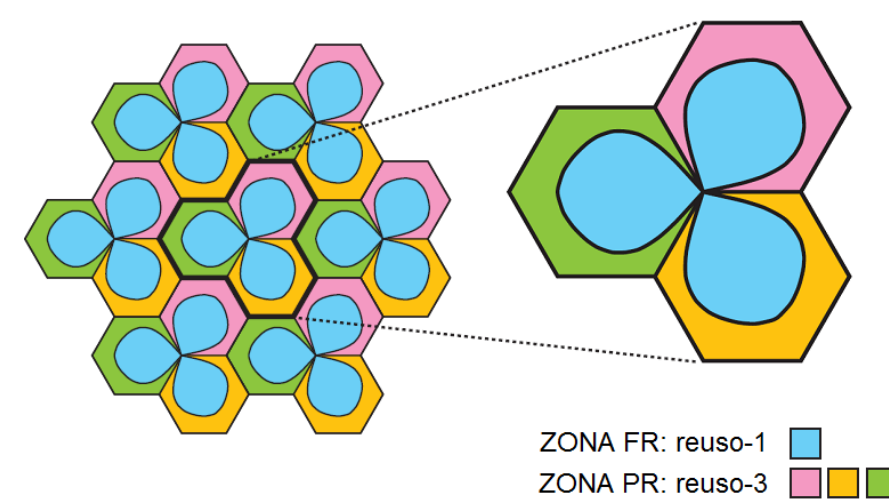

Figura 2. Asignación de frecuencias en las celdas.

Parcial (PR), tres bandas de frecuencia se asignan cíclicamente (reuso-3), mientras que en las zonas de Reuso Completo (FR), se emplea siempre la misma frecuencia (reuso-1) (11).

Para facilitar el diseño de la red se tienen 2 parámetros principales en la configuración de recursos para la zona de Reuso Completo (FR) y Reuso Parcial (PR) los cuales son:

\section{- Umbral de Relación Señal a Ruido más Inter-} ferencia (SINR): denominado $\Gamma_{t h r}$, define el área de la celda asignado a cada zona, mediante este se especifica el punto de SINR en el que se realiza la conmutación entre las zonas de Reuso Completo (FR) y Reuso Parcial (PR), considerando un rango de SINR desde $22.5 \mathrm{~dB}$ hasta $-2 \mathrm{~dB}$, valores que abarcan el máximo valor de potencia presente en la zona centro y el mínimo valor de potencia considerado para un usuario en la zona borde (12). El ancho de banda de SINR $(\Gamma)$ se define en la ecuación [1]:

$$
\Gamma=\frac{G_{\text {antena }} L_{\text {Macro }, 0} P_{T X 0}}{\sigma_{n}^{2}+\sum_{m=1}^{N_{\text {int }}} L_{\text {Macro }, l} P_{T X l}}
$$


Donde:

$G_{\text {antena }}=$ Ganancia de la antena.

$L_{\text {Macro, },}=$ Pérdidas macroscópicas de la señal transmitida.

$P_{T X 0}=$ Potencia de transmisión de la Estación Base.

$L_{\text {Macro,l }}=$ Pérdidas macroscópicas de la señal interferente.

$P_{T X l}=$ Potencia de transmisión de la Estación Base interferente.

$\sigma_{n}^{2}=$ Potencia de ruido promedio por antena receptora.

En la Figura 3, se observan los posibles valores umbrales de SINR que se encuentran dentro del rango previamente establecido y que comprenden todas las posibles sectorizaciones de la celda.

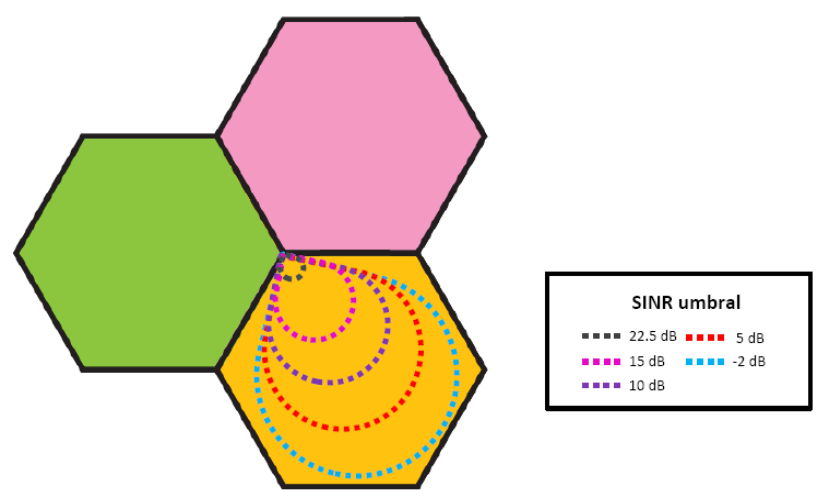

Figura 3. Umbrales de SINR para las zonas de Reuso Completo (FR) y Reuso Parcial (PR).

- Partición del Ancho de Banda (BW, BandWidth): denominado $\beta_{F R}$, determina la banda en frecuencia a ser empleada en la zona de Reuso Completo (FR) y Reuso Parcial (PR), donde las variaciones de $\beta \_$FR van desde $0.01,0.04, \ldots$. Hasta 1, con lo cual se proporciona un número entero de Bloque de Recursos Físicos (PRB, Physical Resource Block) a cada zona (12).

En la Figura 4, se observa como una fracción del Ancho de Banda total $\left(\beta_{F R}\right)$ se asigna a la banda de frecuencia empleada en las zonas de Reuso Completo (FR), representada en color azul, mientras que el Ancho de Banda (BW) restante $\left(1-\beta_{F R}\right)$ es igualmente asignado a cada una de las tres bandas en color rosa, naranja y verde del Reuso Parcial (PR), es decir (1 $\left.-\beta_{F R}\right) / 3$ es asignado a cada zona del borde de la celda (12)

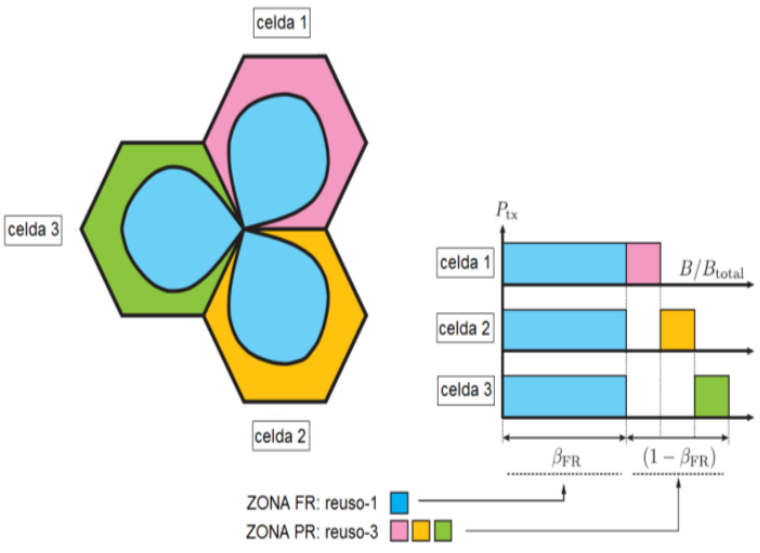

Figura 4. Asignación de Ancho de Banda (BW) en las bandas de frecuencia empleadas.

\section{Configuración de la simulación}

En la Tabla 1, se muestra la lista completa de los parámetros de simulación, donde se tienen los algoritmos de planificación Round Robin y Proportional Fair, aplicados tanto en la zona de Reuso Completo (FR) como de Reuso Parcial (PR).

Considerando el número de valores de $\beta_{F R}$ y $\Gamma_{t h r}$, se realizaron 3366 simulaciones tanto para Round Robin como para Proportional Fair, a través de las cuales se evaluó el desempeño de todas las posibles configuraciones.

\section{RESULTADOS Y DISCUSIÓN}

\section{A. Evaluación de las Configuraciones de FFR}

La evaluación del Throughput de un sistema empleando Reuso de Frecuencia Fraccional (FFR) se realiza por medio de Indicadores Claves de Desempeño (KPI, Key Performance Indicators), más conocidos como: Throughput Medio, Borde y Pico (13). Estos indicadores se derivan de la Función de Probabilidad Acumulada Empírica (ECDF, Empirical Cumulative Density Function) en el Throughput. El indicador Throughput Borde es el resultado de emplear el 5\% de los puntos de la Función de Distribución Acumulada Empírica (ECDF) y el Throughput Pico de emplear el 95\% (14), lo anterior se interpreta como el desempeño de un Equipo de Usuario en el borde y en el centro de la celda, respectivamente (15).

En la Figura 5, se muestran de izquierda a derecha los resultados del Throughput Medio, Pico y Borde alcanzados por un sistema LTE con Reuso de Frecuencia Fraccional (FFR) y el algoritmo de planificación 
Tabla 1. Parámetros de simulación empleados para las simulaciones de FFR en LTE.

\begin{tabular}{|c|c|}
\hline Frecuencia & $2 \mathrm{GHz}$ \\
\hline Ancho de Banda Total & $20 \mathrm{MHz}(100 \mathrm{PRBs})$ \\
\hline Distancia entre eNodeBs & $500 \mathrm{~m}$ \\
\hline Número eNBs considerados & 7 sitios (21 celdas) \\
\hline Numero de UEs por celda & 30 \\
\hline Pérdida min de acoplamiento & 70dB Zona Urbana \\
\hline \multicolumn{2}{|l|}{ (REC TS 36.942) } \\
\hline Modelo de pérdida de propagación macroscópica & $\begin{array}{l}\text { Zona Urbana } \\
\mathrm{L}=40\left(1-4 * 10^{-3} \mathrm{Dhb}\right) \log 10(\mathrm{R}) 18 \log 10(\mathrm{Dhb}) \\
21 \log 10 \text { (f) } 80 \mathrm{~dB}\end{array}$ \\
\hline Desvanecimiento por Sombra & Ninguno \\
\hline Tipo de Antena & kathrein 742212 \\
\hline Arreglo de Antenas & $1 \mathrm{x} 1$ \\
\hline Potencia de transmisión & $40 \mathrm{w}$ \\
\hline Ganancia Antena & $15 \mathrm{dBi}$ \\
\hline Frecuencia Antena & $2140 \mathrm{MHz}$ \\
\hline Altura del Transmisor & $20 \mathrm{~m}$ \\
\hline Altura del Receptor & $1.5 \mathrm{~m}$ \\
\hline Modelo de Canal & Winner fase II \\
\hline Velocidad de los UEs & $1,38 \mathrm{~m} / \mathrm{s}$ (ITU Peatonal A) \\
\hline Figura de Ruido & $7 \mathrm{~dB}$ \\
\hline Densidad de ruido térmico & $-174 \mathrm{~dB} / \mathrm{Hz}$ \\
\hline Rango de SINR Umbral ( $\Gamma_{-}$thr $)$ & $-2: 0.25: 22.5$ (99 valores) \\
\hline Rango de la tasa de $\beta$ FR & 0.01:0.03:1 (34 valores) \\
\hline Número total de simulaciones & 3366 \\
\hline Modelo de Tráfico & Full Buffer \\
\hline Modelado Receptor & Zero Forcing \\
\hline \multirow[t]{2}{*}{ Algoritmos de Planificación } & -Round Robin \\
\hline & -Proportional Fair \\
\hline
\end{tabular}


Proportional Fair. Para obtener las gráficas de desempeño de un Equipo de Usuario (UE), se variaron los parámetros $\beta_{F R}$ en el eje x y $\Gamma_{t h r}$ en el eje y (12).

Como se puede observar en la Figura 5, cada punto de color representa el nivel de Throughput experimentado por un Equipo de Usuario (UE) en determinada zona de la celda. Si el usuario se encuentra en la zona centro de la celda (gráfica del centro), la máxima ganancia del Throughput que alcanzará será de $25 \mathrm{Mbps}$ en comparación con el reuso de uno, en el intervalo de $\Gamma_{t h r}=18 \mathrm{~dB} \operatorname{con} \beta_{F R}=1$ y $\Gamma_{t h r}=22.5 \mathrm{~dB}$ con $\beta_{F R}=0.9$. Pero si el usuario se encuentra en la zona borde de celda (gráfica del lado derecho), la máxima ganancia será de 0.7 Mbps para el intervalo de $\Gamma_{t h r}=10 \mathrm{~dB} \operatorname{con} \beta_{F R}=0.30$ y $\Gamma_{t h r}=15 \mathrm{~dB} \operatorname{con} \beta_{F R}=0.25$. Del mismo modo, se observa que el máximo Throughput Medio (gráfica del lado izquierdo) alcanzado es igual a $2.5 \mathrm{Mbps}$ para el intervalo entre $\Gamma_{t h r}=15 \mathrm{~dB} \operatorname{con} \beta_{F R}=1$ y $\Gamma_{t h r}=20 \mathrm{~dB} \operatorname{con} \beta_{F R}=0.9$.

En la Figura 6, se modela en términos porcentuales (\%) la ganancia del Throughput obtenida en las diferentes zonas de la celda al emplear el Reuso de Frecuencia Fraccional (FFR) en LTE. A modo de ejemplo, si se configura el esquema de reuso con $\beta \_F R=0.30$ y $\Gamma_{-}$thr $=10 \mathrm{~dB}$, se obtienen los siguientes resultados de desempeño de un Equipo de Usuario (UE) (12):

- Throughput Medio: igual a 1.3 Mbps, equivalente a una ganancia del 5\% en relación con el reuso de uno (ver Figura 5 y Figura 6 del lado izquierdo).

- Throughput Pico: igual a 4 Mbps similar al 70\% de ganancia (ver Figura 5 y Figura 6 del centro).

- Throughput Borde: igual a $0.7 \mathrm{Mbps}$, semejante al 45\% de ganancia (ver Figura 5 y Figura 6 del lado derecho).
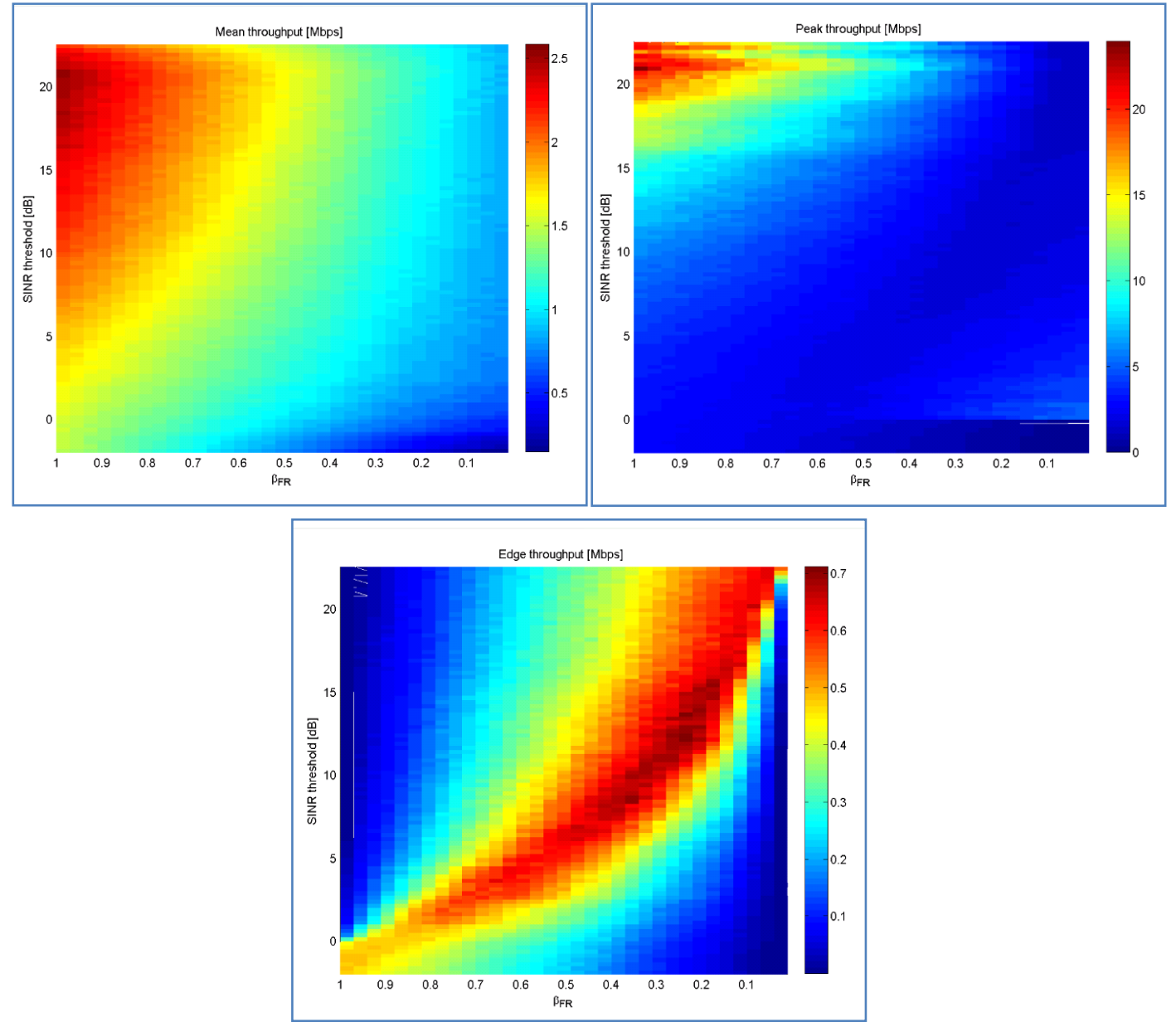

Figura 5. Resultados de Simulación del Throughput Medio, Pico y Borde (Mbps) para un Sistema LTE con FFR y Proportional Fair. 

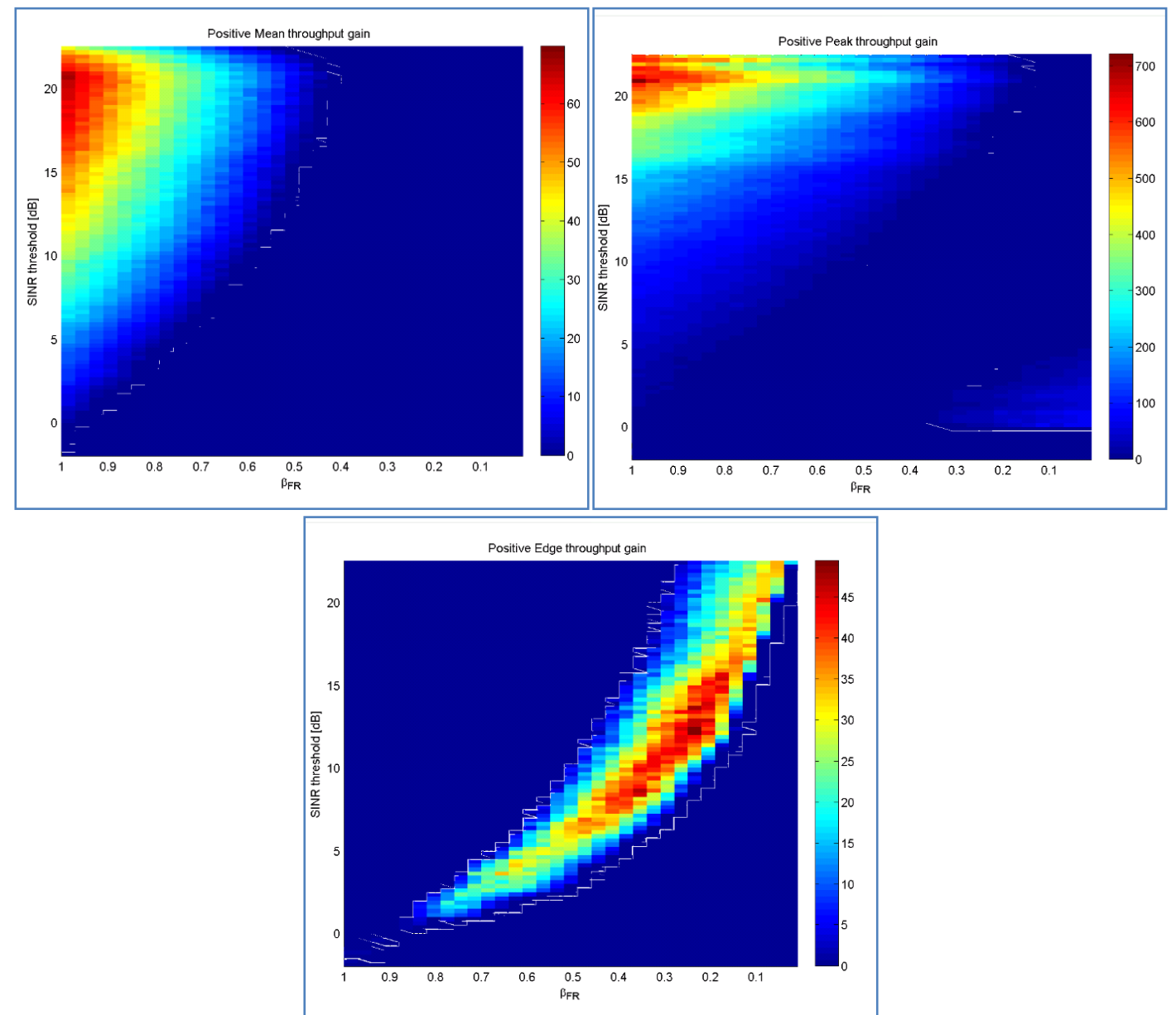

Figura 6. Resultados de Simulación de la Ganancia Positiva del Throughput Medio, Pico y Borde (\%) para un Sistema LTE con FFR y Proportional Fair.

\section{B. Evaluación de la Capacidad}

Con el fin de evaluar la capacidad de un sistema LTE al emplear la técnica de Reuso de Frecuencia Fraccional (FFR), en los siguientes resultados se encuentra la distribución de Throughput y Eficiencia Espectral en función del Ancho de Banda (BW) de la SINR para los Equipos de Usuario (UE) de un sistema LTE y los diferentes esquemas de reuso. Estos resultados se presentan de dos formas (12):

- En curvas de Función de Probabilidad Acumulada Empírica (ECDF).

- En diagramas de dispersión.

Es importante resaltar que estos resultados representan la ganancia en Throughput y eficiencia espectral obtenidos al implementar la técnica de Reuso de Frecuencia Fraccional (FFR) en LTE.

Las curvas de Función de Probabilidad Acumulada Empírica (ECDF) se observan en la Figura 7, ésta muestra los resultados de Throughput y Eficiencia Es- pectral para el escenario con $\Gamma_{t h r}=10 \mathrm{~dB}$ y $\beta_{F R}=0.75$. A la izquierda, la $\mathrm{F}(\mathrm{x})$ que está en función del Throughput medio de los Equipos de Usuarios (UEs) [Mbps], indica el porcentaje de usuarios que experimentan ciertos niveles de Throughput. Del mismo modo, a la derecha, se encuentra la $\mathrm{F}(\mathrm{x})$ en función de la Eficiencia espectral media de los Equipos de Usuarios (UEs) [bit/cu].

Por ejemplo, la curva del lado izquierdo señala que aproximadamente el 58\% de los usuarios tienen bajos niveles de Throughput, sin embargo el otro 42\% al tener niveles más altos, impulsan a que el Throughput medio de la celda aumente y genere ganancia en comparación con un sistema sin reuso (12).

Para el mismo escenario $\left(\Gamma_{t h r}=10 \mathrm{~dB}\right.$ y $\beta_{F R}=0.75$.), los diagramas de dispersión se presentan en la Figura 8; en ella se observa que en $\Gamma_{t h r}=10 \mathrm{~dB}$, tanto para los resultados del Throughput como de Eficiencia Espectral, se delimita la Zona de Reuso Completo (FR) y la Zona de Reuso Parcial (PR). La zona FR se denomina 


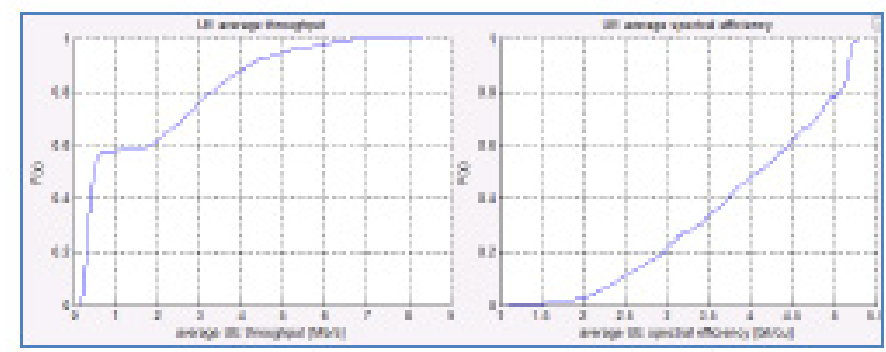

Figura 7. Curvas de Función de Probabilidad Acumulada Emperica (ECDF).

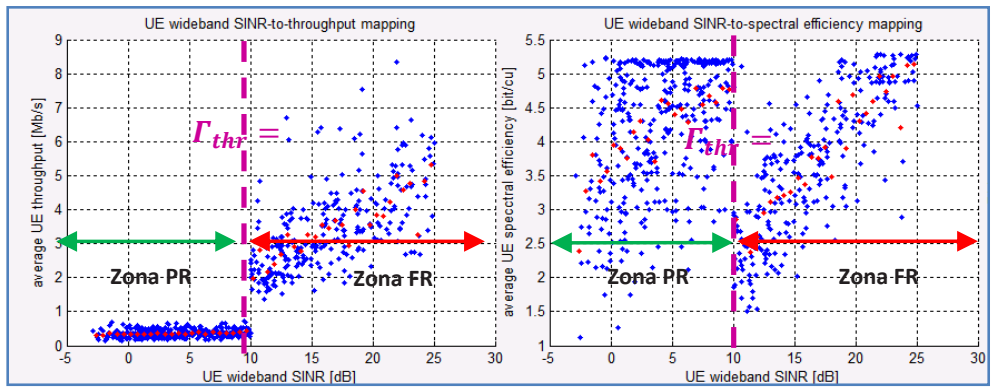

Figura 8. Diagramas de Dispersión.

zona centro de celda por tener los Equipos de Usuarios (UE) con los mejores niveles de SINR, mientras que la Zona PR se conoce como zona borde al abarcar usuarios con Relación Señal a Ruido más Interferencia (SINR) bajos.

En la Figura 8 a la izquierda, se puede ver que a pesar de emplear la técnica de Reuso de Frecuencia Fraccional (FFR), los Equipos de Usuario (UE) de la Zona PR presentan niveles de Throughput promedio muy bajos en relación con los de la Zona FR, por lo que es de gran importancia establecer el mejor escenario para el cual la técnica muestra los mejores resultados de Throughput y eficiencia espectral en el borde de celda.

La Figura 9, muestra las curvas de Función de Probabilidad Acumulada Empírica (ECDF) y los diagramas de dispersión del algoritmo de planificación Proportional Fair para $\boldsymbol{\Gamma}_{t h r}=10 \mathrm{~dB}$ con $\boldsymbol{\beta}_{\boldsymbol{F R}}=0.25$, que es la mejor configuración en la implementación del reuso fraccional. Los puntos rojos del diagrama de dispersión del Throughput indican que la tendencia de la gráfica se mantiene en un rango entre $0.5 \mathrm{Mbps}$ y 1 Mbps para la zona PR y entre 0.7 Mbps y $1.7 \mathrm{Mbps}$ para la zona FR, lo que exhibe un comportamiento equitativo entre las dos zonas y demuestra la mejora significativa del Throughput en la zona PR. Además, al analizar la Función de Probabilidad Acumulada Empírica (ECDF) en el Throughput, se observa que la probabilidad de que los usuarios alcancen una ganancia por encima de $1 \mathrm{Mbps}$ es del 57\%, mientras que para otras configuraciones es menor.

Por otra parte, los resultados de la eficiencia espectral son los más altos en relación con otras configuraciones al logar que el 78\% de los Equipos de Usuario (UE) alcancen valores superiores a 3 [bit/cu], ya que la tendencia de sus diagramas de dispersión presentan comportamientos curvilíneos entre 3 [bit/cu] y 5 [bit/ $\mathrm{cu}$ en la zona borde y entre $2[\mathrm{bit} / \mathrm{cu}]$ y $5[\mathrm{bit} / \mathrm{cu}]$ en la zona centro.

\section{Evaluación probabilística del Throughput y la eficiencia espectral}

La evaluación probabilística se realiza través del método Montecarlo (17) para analizar el comportamiento de la red y corroborar cuál es el mejor escenario de la técnica de Reuso de Frecuencia Fraccional (FFR).

En total se realizaron 63000 simulaciones obtenidas de repetir 100 veces un escenario de simulación con 21 celdas y 30 usuarios por cada una, para determinar una muestra suficientemente grande que permitiera obtener una tendencia estadística de cada escenario.

Para este sistema estocástico se emplearon los escenarios de prueba que se muestran en la Tabla 2, con el fin de analizar estadísticamente el Throughput y la 

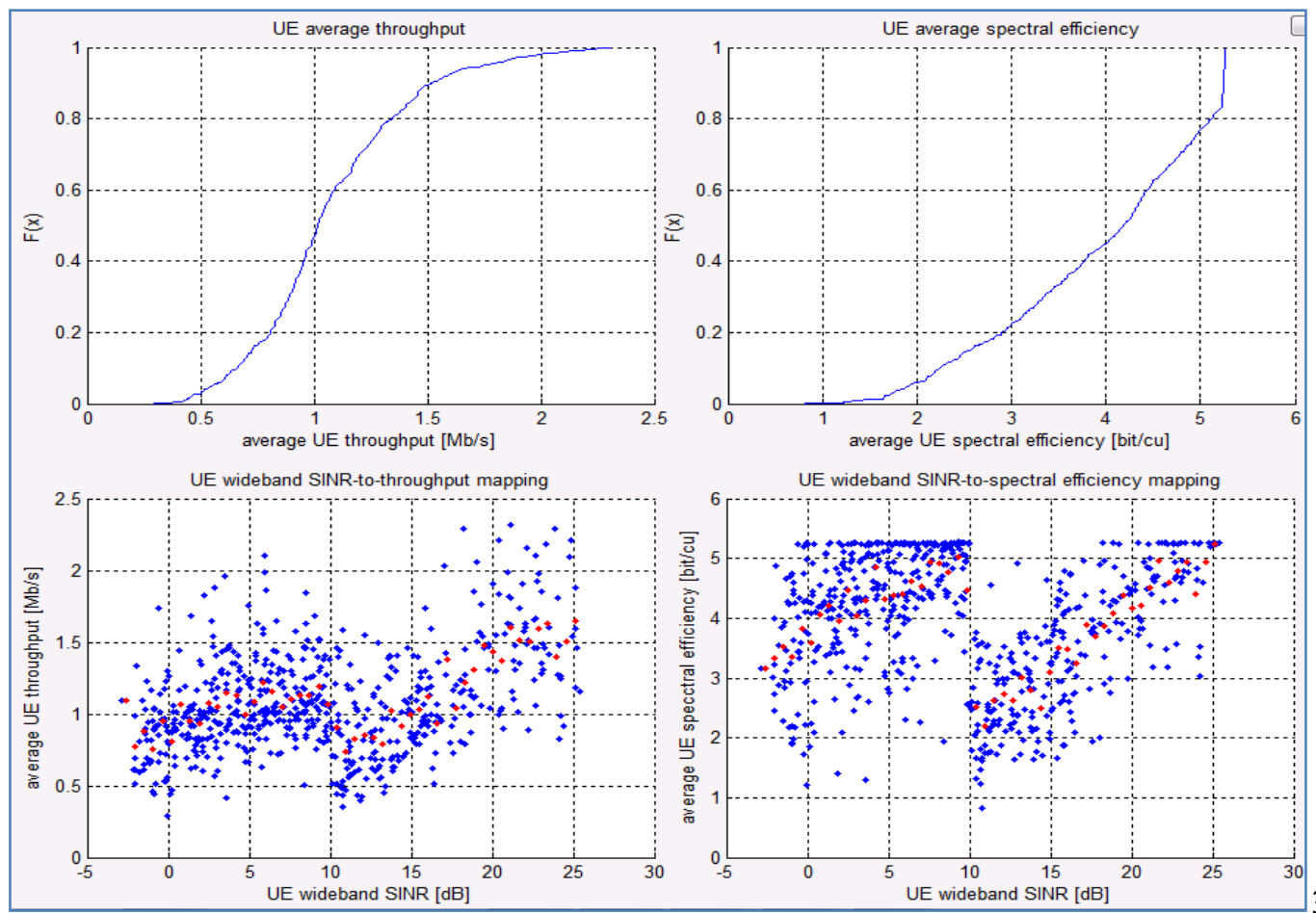

Figura 9. Curvas de Función de Probabilidad Acumulada Empírica (ECDF) y Diagramas de Dispersión para el Escenario $\boldsymbol{\Gamma}_{t h \boldsymbol{r}}=10 \mathrm{~dB}$ para $\boldsymbol{\beta}_{\boldsymbol{F}}=0.25$.

eficiencia espectral y por ende la Interferencia InterCelda (ICI), de tal forma que fuese posible analizar el comportamiento de la red con y sin la técnica de mitigación.

Tabla 2. Escenarios de prueba más representativos.

\begin{tabular}{cccc}
\hline \multicolumn{3}{c}{ THROUGHPUT } \\
\hline ESCENARIO & PROMEDIO & VARIANZA \\
\hline REUSO 1 & 1,07796372 & 1,35133971 \\
REUSO 3 & 0,73378522 & 0,27501987 \\
& SINR $=10, \beta_{F R}=0.25$ & 0,73646531 & 0,256027628 \\
& $\mathrm{SINR}=10, \beta_{F R}=0.30$ & 0,81464653 & 0,3041054 \\
& $\mathrm{SINR}=10, \beta_{F R}=0.50$ & 1,01341605 & 1,04954534 \\
FFR & $\mathrm{SINR}=10, \beta_{F R}=0.75$ & 1,25294785 & 2,61651577 \\
& $\mathrm{SINR}=15, \beta_{F R}=0.25$ & 0,88679546 & 0,67270858
\end{tabular}

Las Tablas 3 y 4 presentan los cálculos probabilísticos de las 63000 simulaciones de cada escenario con el planificador Proportional Fair.

Las Figuras 10 y 11 presentan la distribución normal o campanas de gauss (18) obtenidas de las Tablas 3 y 4 respectivamente, en donde se observan las funciones de densidad de probabilidad para el Throughput y para la eficiencia espectral con Proportional Fair.
Tabla 3. Valores de media y varianza del Throughput para cada escenario con Proportional Fair.

\begin{tabular}{cccc}
\hline \multicolumn{3}{c}{ THROUGHPUT } \\
\hline \multicolumn{1}{c}{ ESCENARIO } & PROMEDIO & VARIANZA \\
\hline REUSO 1 & 1,07796372 & 1,35133971 \\
REUSO 3 & 0,73378522 & 0,27501987 \\
& SINR $=10, \beta_{F R}=0.25$ & $\mathbf{0 , 7 3 6 4 6 5 3 1}$ & $\mathbf{0 , 2 5 6 0 2 7 6 2 8}$ \\
& $\operatorname{SINR}=10, \beta_{F R}=0.30$ & $\mathbf{0 , 8 1 4 6 4 6 5 3}$ & 0,3041054 \\
FFR & $\mathrm{SINR}=10, \beta_{F R}=0.50$ & 1,01341605 & 1,04954534 \\
& $\operatorname{SINR}=10, \beta_{F R}=0.75$ & 1,25294785 & 2,61651577 \\
& $\operatorname{SINR}=15, \beta_{F R}=0.25$ & 0,88679546 & 0,67270858 \\
& $\operatorname{SINR}=15, \beta_{F R}=0.50$ & 1,16595773 & 3,00119565 \\
& $\operatorname{SINR}=15, \beta_{F R}=0.75$ & 1,51616762 & 7,78248664 \\
\hline
\end{tabular}

Tabla 4. Valores de media y varianza de la eficiencia espectral para cada escenario con Proportional Fair.

\begin{tabular}{cccc}
\hline \multicolumn{3}{c}{ EFICIENCIA ESPECTRAL } \\
\hline \multicolumn{2}{c}{ ESCENARIO } & PROMEDIO & VARIANZA \\
\hline REUSO 1 & 1,85592079 & 2,43699462 \\
REUSO 3 & 4,20042938 & 2,02453207 \\
& SINR= 10, $\beta_{F R}=0.25$ & 4,09462948 & 2,10841776 \\
& SINR= 10, $\beta_{F R}=0.30$ & 3,28252883 & 2,56230368 \\
& FINR $=10, \beta_{F R}=0.50$ & 3,32562983 & 2,53510472 \\
& $\operatorname{SINR}=10, \beta_{F R}=0.75$ & 3,24619983 & 2,86130975 \\
& $\operatorname{SINR}=15, \beta_{F R}=0.25$ & 3,76015987 & 2,40330362 \\
& $\operatorname{SINR}=15, \beta_{F R}=0.50$ & 3,74291787 & 2,38915615 \\
& $\operatorname{SINR}=15, \beta_{F R}=0.75$ & 3,87595186 & 3,37472105 \\
\hline
\end{tabular}




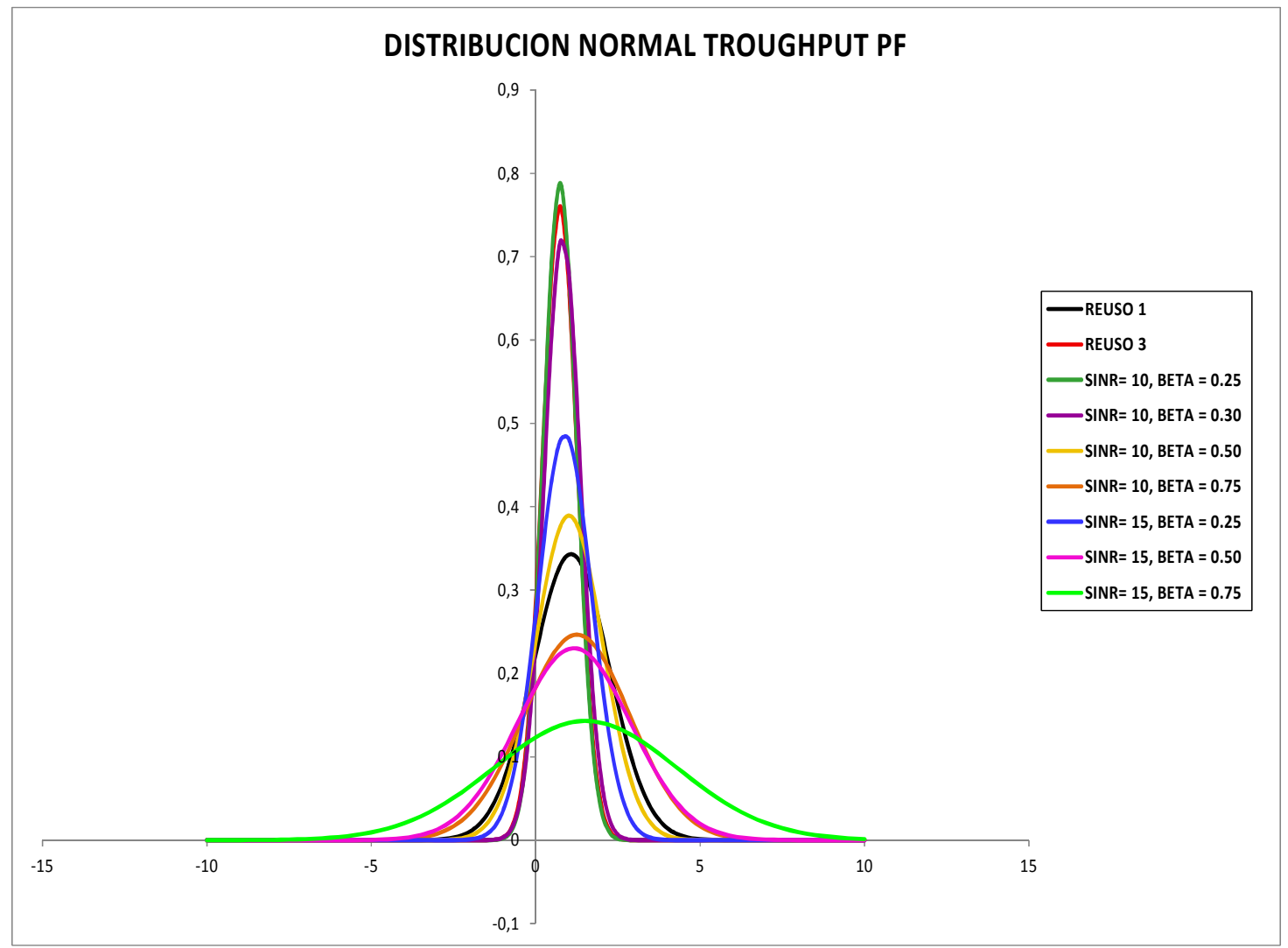

Figura 10. Distribución normal del Throughput en cada escenario para Proportional Fair.

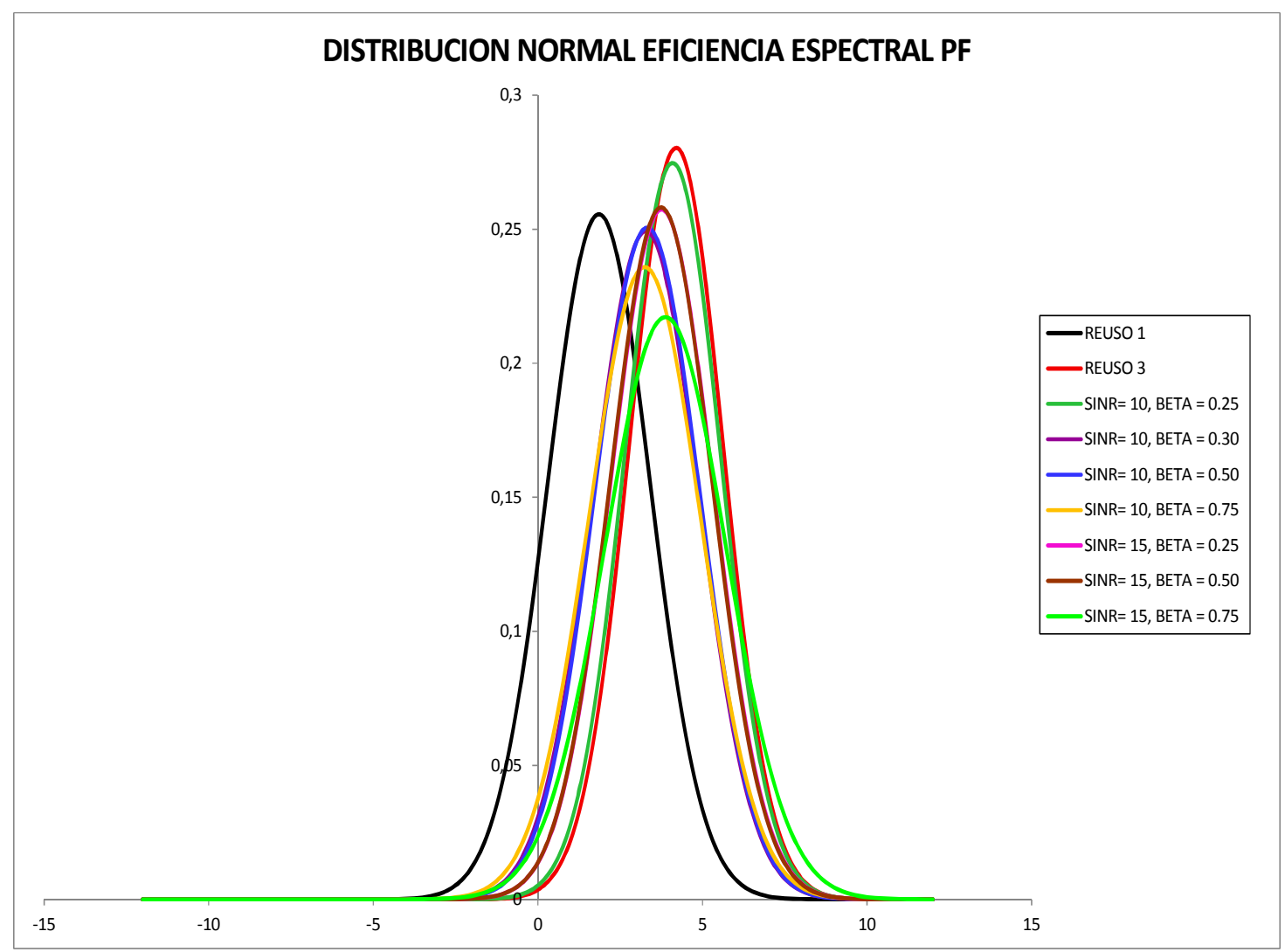

Figura 11. Distribución normal de la eficiencia espectral en cada escenario para Proportional Fair. 
Analizando la Figura 10, se observa que para los casos de Reuso de Frecuencia Fraccional (FFR) hay una alta probabilidad de ocurrencia de que el Throughput tome un valor cercano a la media de cada escenario, de esta forma en la Tabla 3 se definen en color azul los mejores escenarios, donde el valor medio se mantiene y la varianza disminuye en comparación con el reuso 1 y reuso 3 , con lo cual garantiza que tanto usuarios del borde como del centro tengan un desempeño similar, permitiendo que sus velocidades sean aproximadamente iguales.

En la Figura 11, se observan los resultados correspondientes a la eficiencia espectral de la celda, donde se ve claramente que para el Reuso de Frecuencia Fraccional (FFR), las curvas se desplazan hacia la derecha garantizando que la media aumente y se mantenga casi igual a la obtenida con el reuso 3 .

Siendo así, en la Figura 11 se observan en color rojo los mejores resultados de eficiencia espectral para usuarios con SINR baja y alta.

De esta forma, se puede decir que los altos valores de Throughput y eficiencia espectral en los usuarios borde y centro, se debe a que la Interferencia Inter-Celda (ICI) disminuye y permite que tengan una calidad del servicio aceptable.

\section{CONCLUSIÓN}

El Reuso de Frecuencia Fraccional (FFR) en redes LTE, favorece el desempeño de los usuarios del borde, ya que permite mitigar la Interferencia Inter-Celda (ICI) al garantizar que las tasas de Throughput dentro de la celda sean equitativas para todos los usuarios y la eficiencia espectral mejore en el borde y se mantenga en el centro.

Los escenarios propuestos junto con el uso de planificadores, permiten tener variedad de opciones en el diseño de las redes que mejoran el desempeño del sistema, dado que la calidad del servicio se mantiene por igual para todos los usuarios.

La configuración $\boldsymbol{\Gamma}_{t h r}=10 \mathrm{~dB}$ con $\boldsymbol{\beta}_{F R}=0.25$, es el mejor escenario para mitigar la Interferencia Inter-Celda de un sistema SISO en LTE, ya que genera altos niveles de Throughput y eficiencia espectral en el borde de la celda.
El simulador LTE de Viena a nivel de sistema, es de gran ayuda en el análisis del desempeño de nuevas tecnologías, ya que al ser gratuito y de uso académico, favorece la investigación en ambientes universitarios.

\section{AGRADECIMIENTOS}

Los autores agradecen a la Universidad del Cauca, la Facultad de Ingeniería Electrónica y Telecomunicaciones, el Departamento de Telecomunicaciones, a su grupo de investigación: Grupo I+D Nuevas Tecnologías en Telecomunicaciones (GNTT) por el soporte brindado para el desarrollo de este trabajo de grado. Se agradece especialmente al Instituto de Comunicaciones e Ingeniería de Radio-Frecuencia de la Universidad de Viena, Austria por permitir el uso de la herramienta de simulación para el desarrollo de las simulaciones.

Del mismo modo se expresa un especial agradecimiento al ingeniero Mg. Víctor Manuel Quintero Flórez y al ingeniero Mauricio Ramírez por sus aportes en este trabajo.

\section{BIBLIOGRAFÍA}

1. Khan, F. LTE for 4G Mobile Broadband. Primera edición ed. New York: Cambridge University Press; 2009.

2. Diaz, E. J. "Análisis de las Estrategias de Gestión del Espectro Radioeléctrico en un Sistema LTE con la herramienta Atoll". Tesis de Grado. Universidad Politecnica de Catalunya. 2012.

3. Rizwan G, Raymond K. "Fractional Frequency Reuse and Interference Suppression for OFDMA Networks". En Modeling and Optimization in Mobile, Ad Hoc and Wireless Networks (WiOpt), Proceedings of the 8th International Symposium on ( 273 - 277). Avignon, France. 2010.

4. Comes, R., Alvarez, F. B., Casadevall, F., Ferrus, R., Perez, Romero, J. y Sallent, O. LTE: nuevas tendencias en comunicaciones móviles: Fundación Vodafone España. 2010.

5. Lee, P. y Lee, T. "Interference Management in LTE Femtocell Systems Using Fractional Frequency Reuse". En Advanced Communication Technology (ICACT), The 12th International Conference on (Volume:2 ) 2010; 1047 - 1051.

6. Tiwari, B. 2010. "Enabling Reuse 1 in 4G Networks". Consultado Octubre 10, 2013, en http://www.beyond4g.org/.

7. Bilios, D. y Bouras, C. "Optimization of Fractional Frequency Reuse in Long Term Evolution Networks". En Wireless Communications and Networking Conference (WCNC),2012 IEEE 2012; 1853 - 1857. Shanghai. 
8. Chang, R., Tao, Z., Zhang, J. y Kuo, J. "A Graph Approach to Dynamic Fractional Frequency Reuse (FFR) in Multi-Cell OFDMA Networks". En ICC'09 Proceedings of the 2009 IEEE international conference on Communications. 2009; 3993-3998.

9. Saquib, N., Hossain, E. y Kim, D. "Fractional Frequency Reuse for Interference Management in LTEAdvanced HetNets". En Wireless Communications, IEEE (Volume:20, Issue: 2 ) 2013; 113 - 122.

10. Ikuno, J., Wrulich, M. y Rupp, M. "System level simulation of LTE networks". En Vehicular Technology Conference (VTC 2010-Spring), 2010 IEEE 71st (1- 5).Taipei.

11. Krasniqi, B., Wrulich, M. y Mecklenbrauker, C. "Network-load Dependent Partial Frequency Reuse for LTE". En Communications and Information Technology, 2009. ISCIT 2009. 9th International Symposium on 2009; 672 - 676. Icheon.

12. Ikuno, J. C., Taranetz, M. y Rupp, M. "A Fairness-Based Performance Evaluation of Fractional Frequency Reuse in LTE”. En 17th International ITG Workshop on Smart Antennas. 2013.

13. Taranetz, M. y Ikuno, J. C. "Capacity Density Optimization by Fractional Frequency Partitioning”. En Annual Asilomar Conference on Signals, Systems, and Computers. California. 2011.

14. Andrews, J. G., Illsoo, S., Ganti, R. K. y Ghosh. "Comparison of Fractional Frequency Reuse Approaches in the OFDMA Cellular Downlink". En IEEE Global Telecommunications Conference 2011; 1-5. Miami.

15. Chen, L. y Yuan, D. "Generalized Frequency Reuse Schemes for OFDMA Networks: Optimization and Comparison". En Vehicular Technology Conference (VTC 2010-Spring), 2010 IEEE 71st (1-5). Taipei. 2010.

16. Colom, J., Wrulich, M. y Rupp, M. 2010. "LTE Downlink System Level Simulator". Consultado 2014. en: http://www.nt.tuwien.ac.at/research/mobile-communications/lte-downlink-system-level-simulator/.

17. Santos del Cerro, J. Historia de la probabilidad y la estadística. III. Primera ed. Garcia F, editor. Madrid: Delta Publicaciones. 2006.

18. Condor, I. Teoria de la Probabilidad y Aplicaciones Estadísticas. Primera ed. Condor I, editor. 